\title{
Are we facing an epidemic of smoking-related cancers and treatment complications in transitional countries?
}

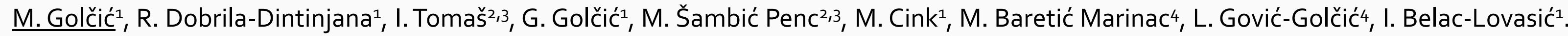

${ }^{1}$ Clinical Hospital Center Rijeka, Department for Radiotherapy and Oncology, Rijeka, Croatia.

${ }^{2}$ Clinical Hospital Center Osijeka, Radiotherapy and oncology, Osijek, Croatia.

3University of Osijek, School of Medicine, Osijek, Croatia.

4GP Office, Family Medicine, Rijeka, Croatia.

\section{INTRODUCTION}

Cigarette smoking is one of the most researched factors that cause cancer and is also associated with a higher total symptom burden during cancer treatment. Transitional countries, such as Croatia, exhibit a large number of smokers (around 30\%), however, not much is known about the smoking habits of our patients during cancer treatment.

\section{METHODS}

The study was cross-sectional research using a simple questionnaire in two clinical hospital centres in Croatia and involved 441 cancer patients with history of smoking, who were undergoing active treatment.

Number of smokers in Croatia, both genders, 2015

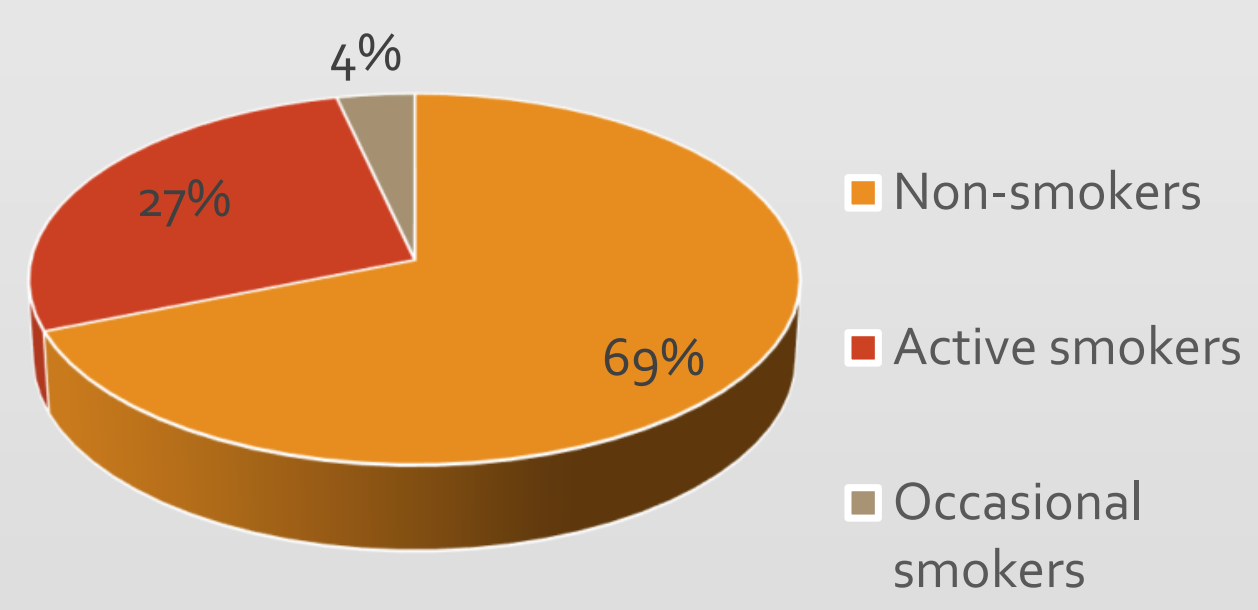

Smoking habits based on median age $(\mathrm{N}=686)$

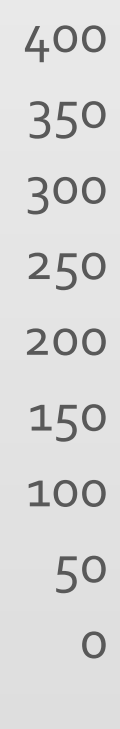

350
300
250
200
150
100
50
0

\section{RESULTS}

The median age of cancer patients was 62. Patients younger than the median age were more often previous smokers $(72 \%$ vs. 56\%), smokers at the time of cancer diagnosis $(63 \%$ vs. $39 \%)$ and during the cancer treatment ( $52 \%$ vs $35 \%$ ). Before cancer diagnosis, > 10 cigarettes a day were smoked by around $56 \%$ of younger patients and only $39 \%$ of older patients, although the ratio reversed during the cancer treatment ( $34 \%$ vs $38 \%$ ).

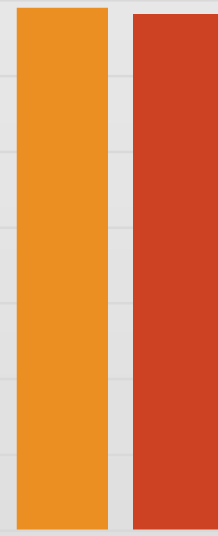

All patients

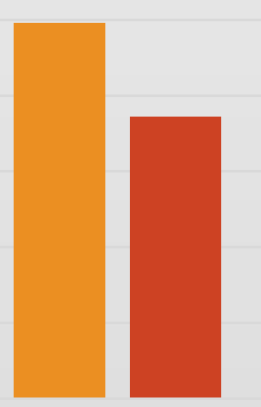

Ever smoked

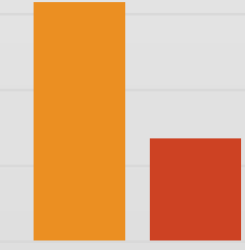

Smoked during diagnosis

62 or younger $\quad 63$ and olde

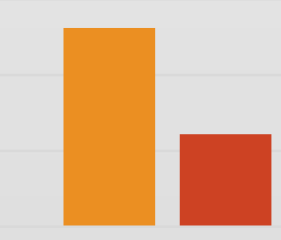

Smokes during treatment
Number of cigarettes smoked per day, based on median age

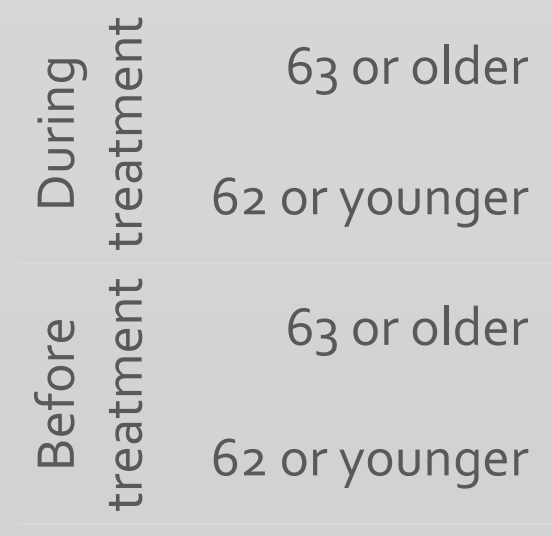

0

100

200

300

400

10 or less $\square$ more than 10

\section{CONCLUSION}

There is a significantly higher percentage of younger smokers both before, during and after cancer diagnosis. Also, younger patients are shown to smoke a greater number of cigarettes before cancer diagnosis. We can expect a dramatic rise in the incidence of smoking-related cancers and treatment complications as the younger patients approach the median age of cancer incidence in Croatia. The data shows that urgent anti-smoking public health campaigns are of utmost importance for transitional countries such as Croatia. 